\title{
LA ALIANZA DEL PACÍFICO: UNA ESTRATEGIA EFECTIVA PARA FORTALECER LA RELACIÓN COMERCIAL CON ASIA-PACÍFICO'
}

\section{MARÍA CECILIA PÉREZ APONTE}

- Profesora de Integración Económica Internacional

\section{ELBA ROO SUPERLANO}

- Profesora de Integración Económica Internacional Facultad de Negocios, UPC

\section{RESUMEN}

Este ensayo tiene por objeto analizar la vinculación entre los compromisos asumidos en el marco del Acuerdo de Asociación Transpacífico (TPP) y su influencia en la profundización de las relaciones peruanas en el marco de la Alianza del Pacífico (AP). Efectivamente, ambos acuerdos contienen compromisos similares en materia de comercio de bienes y servicios, por lo que vincular la relación entre ambos acuerdos merece especial atención en la medida en constituyen, desde la perspectiva de las autoras, punto de partida para la generación de nuevos acuerdos comerciales bilaterales, así como multilaterales.

Palabras clave: Acuerdos comerciales, Alianza del Pacífico, Integración comercial

\section{ABSTRACT}

The purpose of this paper is to analyze the link between the commitments made under the Trans Pacific Partnership Agreement (TPPA) and its influence on the deepening of Peruvian relations within the framework of the Pacific Alliance (PA). Given the fact that both agreements contain similar commitments on trade in goods and services, linking the relationship between them deserves special attention, since they constitute, from the authors' perspective, the starting point for the generation of new bilateral and multilateral agreements.

Key words: Trade agreements, Pacific Alliance, Business integration

1. UPC Review of Global Management, Volumen 3, Número 1, junio 2017
Este ensayo tiene por objeto analizar la vinculación entre los compromisos asumidos en el marco del Acuerdo de Asociación Transpacífico (TPP, por sus siglas en inglés) y su influencia en la profundización de las relaciones peruanas en cuanto a la Alianza del Pacífico (AP). En efecto, tanto el uno como la otra muestran características similares en lo que concierne a la forma de abordar temas como origen, servicios, inversiones, compras gubernamentales y los beneficios para la pequeña y la mediana empresa (pyme), entre otros. Estudiar el vínculo entre ambos acuerdos tiene una importancia especial, porque estos tratados no solo facilitan las relaciones económicas entre el Perú y otros países de interés comercial en el marco del Foro de Cooperación Económica Asia-Pacífico (APEC, por sus siglas en inglés), sino que, adicionalmente, permiten extraer las características del TPP como fuente de información estratégica para ser capitalizada en las negociaciones bilaterales y multilaterales en el futuro,

Desde 2010, el Perú, como parte de su política comercial, ha destinado notables esfuerzos y recursos a la negociación del TPP. Finalmente, el acuerdo fue suscrito por los ministros de Comercio Exterior en febrero de 2016. Sin embargo, en enero de 2017, inmediatamente después de la asunción al poder de Donald Trump como presidente de Estados Unidos, la comunidad internacional fue sorprendida por su decisión de retirar al país del norte del TPP.

Sin duda, este escenario ha planteado una redefinición de la estrategia comercial que emprenderá el Perú para fortalecer su posición en Asia-Pacífico y establecer el nuevo horizonte comercial del país. Mientras este panorama se define, la AP emerge como un escenario clave de integración para fortalecer estratégicamente su capacidad productiva a una escala global y de inserción a mercados, el cual requiere de la activación y el aprovechamiento de las cadenas regionales de valor. Es por ello que, en este ensayo, se desarrolla un análisis comparativo elaborado a partir de los compromisos establecidos en los acuerdos comerciales anteriormente mencionados. 


\section{UN REFERENTE IMPORTANTE DE LA ALIANZA DEL PACÍFICO: EL ACUERDO DE ASOCIACIÓN TRANSPACÍFICD}

El TPP es un acuerdo negociado durante casi siete años por doce países de Asia, América y Oceanía, y tiene por objeto estrechar la relación económica y comercial en el marco del APEC. Las economías participantes, en conjunto, representan el $40 \%$ de la economía mundial y casi un tercio del flujo comercial internacional, e involucra alrededor de 800 millones de habitantes de los doce países miembros. Durante el gobierno del expresidente Barack Obama, el TPP fue un tema central en materia de comercio exterior, y, el 4 de febrero de 2016, este tratado fue suscrito por Australia, Brunéi, Canadá, Chile, Estados Unidos, Japón, Malasia, México, Nueva Zelanda, Perú, Singapur y Vietnam, aunque no había entrado en vigencia por estar aún pendiente la ratificación de las economías miembro².

En la campaña presidencial de Donald Trump, estaba presente la promesa electoral de suspender la aprobación de este acuerdo, en vista de que un importante porcentaje de la población estadounidense, aún hoy en día, considera que podría representar un impacto negativo sobre su empleo y su producción nacional. En particular, Trump es muy crítico de los acuerdos comerciales internacionales, ya que opina que atentan contra las prioridades productivas y comerciales de Estados Unidos ${ }^{3}$. Por su parte, el Gobierno del expresidente Obama apostaba a que el TPP contribuiría a incrementar la presencia de Estados Unidos en Asia, la cual es, desde una perspectiva económica, una de las zonas más dinámicas del mundo. De otro lado, en el ámbito político, permitía mantener la presencia y ejercer influencias en la región, al tener la oportunidad de posicionar reglas comerciales que influyesen regionalmente, lo cual también le ayudaría a contrarrestar la creciente influencia de China en todo Asia-Pacífico (USTR, 2016, pág. 141)

2. El 21 de julio de 2016, en el marco de lo dispuesto por los artículos 56 y 57 de la Constitución Política del Perú, el presidente Ollanta Humala remitió el TPP al Congreso de la República para su aprobación (Perú21, 2016). Cabe indicar que el motivo de esta remisión se basa en el último párrafo del artículo 56 de la mencionada norma, que establece que requieren aprobación del Congreso aquellos tratados que exigen modificación o derogación de alguna ley y los que precisan disposiciones legislativas para su ejecución. En la medida en que el TPP contenía compromisos en materia de propiedad intelectual que requerían aprobación con rango de ley, en particular sobre información no divulgada para productos farmacéuticos biosimilares, se hacía necesaria dicha aprobación (ver artículo 18.51 del TPP)

3. En su página 5, el documento titulado The President's 2017 Trade Policy Agenda, elaborado por el Representante de Comercio de los Estados Unidos (USTR, por sus siglas en inglés), menciona que la actual administración deja en claro que uno de los objetivos priorizados será garantizar un tratamiento justo para agricultores, ganaderos, proveedores de servicios y negocios americanos. En este marco, se estudiarán las barreras que enfrentan las mercancías, los servicios y las inversiones americanas, en particular las no arancelarias. Añade además que, debido a esta política, uno de los objetivos planteados es actualizar los acuerdos comerciales vigentes para reflejar los tiempos de cambio y las condiciones del mercado.
A pesar de la postergación de la aprobación del TPP por Estados Unidos, hoy en día las lecciones que deja este importante acuerdo están plenamente vigentes, y es fundamental extraer algunas características que definitivamente, estarán presentes en los próximos ejercicios de negociación que emprenda el Perú. A continuación, mencionamos algunas de ellas:

- $\quad$ El TPP está totalmente negociado y suscrito. Esto quiere decir que las delegaciones de los doce países lograron concertar las disposiciones jurídicas en 30 capítulos, como resultado de las discusiones de 20 grupos de negociación.

- Inserción de las empresas del bloque en cadenas globales y regionales de valor. El TPP busca promover el desarrollo y el fortalecimiento de la cadena de suministro, para integrar la producción, facilitar el comercio y reducir los costos. A través de las preferencias arancelarias, se procuraría diversificar la oferta exportable, incorporar más tecnología en la producción y en las exportaciones, y elevar los estándares locales de las economías que la integran, hasta armonizarlas entre todos los países miembros. La particularidad más relevante del TPP es la oportunidad que se presenta ante la acumulación regional de origen por parte de las doce economías participantes. Por medio de este mecanismo, el Perú podría importar insumos de cualquier país del bloque a precios competitivos, por aranceles más bajos, e incorporarlos en procesos productivos nacionales para exportar, bien sea productos finales con valor agregado o insumos intermedios, ambos reconocidos como originarios por el resto de los países miembros, lo que brindaría la oportunidad de desarrollar y ampliar las cadenas globales y regionales de valor.

- No obstante lo anterior, hay que destacar que las economías participantes tienen distintos tamaños relativos y composición de sus estructuras productivas. Si bien todos los integrantes del TPP son miembros del APEC y se caracterizan por ser economías abiertas y dinámicas, entre sí mantienen importantes diferencias culturales y geográficas, que se manifestaron en los diversos intereses de sus posiciones negociadoras que se buscaba plasmar en un mismo acuerdo.

- El TPP incluye temas de acuerdos de última generación. Además de contener disposiciones que promueven el intercambio de bienes y servicios y la ampliación de inversiones, también impulsa aspectos más profundos acerca de la participación en procesos de compras públicas, propiedad intelectual y nuevas posibilidades comerciales en exportación de servicios. Igualmente, de los capítulos contenidos en otros acuerdos comerciales, el TPP en particular incorpora compromisos en materia de competitividad y facilitación de negocios, desarrollo e inclusión social.

- Cabe indicar que el TPP incorpora disposiciones sobre empresas de propiedad del Estado y monopolios asignados. Tomando en cuenta que las economías participantes tienen empresas públicas que de alguna manera prestan servicios públicos y otras 
actividades, en el TPP se plasmó un marco de normas sobre las empresas que se dedican principalmente a actividades comerciales, sin que por ello dejen de tener una posición de economías abiertas al respeto de reglas de libre comercio.

- Se incluye, además, un capítulo de pequeñas y medianas empresas (pymes) y desarrollo. Este acuerdo comercial, a propuesta del Perú, abordó dos capítulos de especial importancia para el país. El primero incorpora instrumentos para aprovechar el acuerdo al promover la participación de las pymes en el comercio y garantizar que estas empresas compartan los beneficios que este tratado brinda. El capítulo de desarrollo impulsa el intercambio de experiencias y actividades conjuntas de promoción del crecimiento económico inclusivo entre los países miembros.

- Finalmente, el TPP contiene compromisos en materia de coherencia regulatoria, que buscan generar un entorno regulatorio abierto, justo y predecible para las empresas de la región Asia-Pacífico. Cabe destacar que este tema también se ha negociado en el marco del Acuerdo de Asociación Transatlántica para el Comercio y la Inversión (TTIP) ${ }^{4}$.

Los países miembros de la AP, a excepción de Colombia, han participado activamente en la negociación del TPP y pudieron evaluar la conveniencia y las dificultades de los compromisos suscritos en el acuerdo, de modo particular en cuanto a sus contextos en la relación económica y comercial que se plantea con Asia-Pacífico en la negociación de este acuerdo. Sin lugar a dudas, estos elementos estarán presentes en la estrategia que redefinan en sus próximos pasos, sean a una escala bilateral o a una plurilateral.

\section{¿ES LA ALIANZA DEL PACÍFICO UN ESQUEMA MÁS DE LA INTEGRACIÓN ECONÓMICA DE AMÉRICA LATINA?}

La AP es una iniciativa establecida en abril de 2011 por Chile, Colombia, México y el Perú, y busca alcanzar una integración regional profunda, para avanzar de manera progresiva hacia la libre circulación de bienes, servicios, capitales y personas, y para impulsar las inversiones.

Aunque el Acuerdo Marco de la Alianza del Pacífico se suscribió en junio de 2012 y entró en vigor en julio de 2015 las disposiciones en materia comercial recién se incorporaron en mayo de 2016. La AP mantiene una perspectiva de unificación abierta, que procura convertirse en una plataforma de articulación política y de integración económica y comercial, con proyección al mundo y poniendo énfasis en la región Asia-Pacífico.

El Acuerdo Marco presenta como objetivos fundamentales los siguientes: "a) construir, de manera participativa y consensuada, un área de integración profunda para avanzar progresivamente hacia la libre circulación de bienes, servicios, capitales y personas; b) impulsar un mayor crecimiento, desarrollo y competitividad de las economías de las Partes, con miras a lograr un mayor bienestar, la superación de la desigualdad socioeconómica y la inclusión social de sus habitantes; y c) convertirse en una plataforma de articulación política, de integración económica y comercial, y de proyección al mundo, con especial énfasis en el Asia-Pacífico" (AP, 2012). En este sentido, aborda un amplio conjunto de temas asociados a estas metas, entre los que podemos mencionar asuntos institucionales, comercio e integración, compras públicas, cooperación, cultura, desarrollo minero, educación, estrategia comunicacional, género, innovación, movimiento de personas de negocios y facilitación para el tránsito migratorio, propiedad intelectual, pymes, relacionamiento externo, servicios y capitales, transparencia fiscal, y turismo.

Dentro del contexto del Acuerdo Marco, en febrero de 2014 se suscribió el Protocolo Adicional al Acuerdo Marco de la Alianza del Pacífico, modificado en julio de 2015. En mayo de 2016 entró en vigencia. Este protocolo establece las disciplinas comerciales de los miembros de la AP. Es de destacar que los cuatro países previamente habían celebrado acuerdos comerciales bilaterales entre ellos estableciendo zonas de libre comercio. Por tanto, este protocolo es un aporte para complementar, actualizar, armonizar y profundizar las disposiciones que regían entre sus miembros en dichos acuerdos.

Así, a través de la AP, el Perú busca consolidar, de manera conjunta, las oportunidades que ha procurado en acuerdos comerciales con los socios que conforman esta iniciativa; en particular, las orientadas a la reducción de barreras comerciales, a contribuir en la mejora de la competitividad, a participar en las cadenas internacionales de valor, y a ampliar las posibilidades de atracción de inversión extranjera.

La AP contiene, de esta manera, compromisos con miras a una integración profunda dirigida a crear y a fortalecer las cadenas regionales de valor. Si bien el acelerado avance que ha tenido se explica gracias a los acuerdos de complementación económica vigentes que han suscrito sus miembros en el marco de la Asociación Latinoamericana de Integración (Aladi), el diseño actual en materia comercial se acerca más a los términos y condiciones establecidos en el TPP.

En la actualidad, la AP cuenta con 49 Estados observadores y con la solicitud de Panamá y de Costa Rica para adherirse a ella. Se considera un esquema ambicioso y con amplias posibilidades para alcanzar exitosamente los objetivos trazados. Desde el punto de vista geopolítico, la AP es un actor relevante en la dinámica de configuración de AsiaPacífico y en la iniciativa que se está consolidando, acorde con los planteamientos discutidos en el APEC en América Latina, con una visión estratégica de libre comercio. 


\section{ALGUNAS FORTALEZAS DE LA ALIANZA DEL PACÍFICO}

La AP en América Latina está orientada a establecer alianzas productivas entre los países de la región que a su vez forman parte de la zona denominada Asia-Pacífico. Con esta iniciativa, en el plano comercial, se busca diversificar las matrices productivas regionales, con miras a fortalecer un encadenamiento y a mejorar el valor agregado de las exportaciones. Asimismo, se procura hacer más fuertes las alianzas entre los miembros en áreas como ciencia y tecnología para promover la innovación, lo cual, en conjunto, podría contribuir a profundizar el acceso efectivo de los miembros en los mercados internacionales de bienes y servicios

Particular interés reviste lo relativo a las reglas de origen. En la AP se han establecido normas de origen con una visión de cadena productiva, con medidas específicas más rígidas en aquellos sectores que presentan mayor capacidad de producción de bienes en los países miembros. A través de la simplificación de criterios de requisitos concretos de origen de los integrantes y de la definición de normas claras y unificadas, las reglas de origen se han diseñado para que la producción de bienes finales, con insumos o bienes intermedios de cualquier país, se pueda considerar como bienes nacionales por todos los demás países miembros. De esta manera, se pretende impulsar las cadenas regionales de valor y propiciar una mayor integración productiva, con la meta de incrementar los niveles de competitividad de los cuatro países de la alianza, y de lograr una producción con mayor valor agregado.

De igual modo, los capítulos de obstáculos técnicos al comercio y medidas sanitarias y fitosanitarias de la AP promueven la vigencia de procedimientos y reglas transparentes y previsibles destinadas a evitar obstáculos técnicos innecesarios al comercio, para favorecer en mayor medida el intercambio efectivo de este tipo de bienes, en particular para las industrias agroexportadoras, de cosméticos y farmacéutica. El capítulo de facilitación al comercio cuenta con reglas aduaneras para optimizar el intercambio de bienes, la cooperación entre las autoridades aduaneras y la asistencia mutua (intercambio de información). Las medidas buscan propiciar la transparencia e impulsar tanto el despacho de mercancías con estándares internacionales como la automatización de información. Así, se podrían reducir costos y tiempos en las operaciones comerciales y, de esa manera, mejorar la competitividad.

Cabe mencionar que el Protocolo Adicional al Acuerdo Marco incluye compromisos en materia de coherencia regulatoria en el capítulo 15bis, denominado "Mejora regulatoria", que considera que "la mejora regulatoria se logra a través del establecimiento e implementación sistemática de herramientas como la transparencia y la consulta pública, la revisión y medición ex ante y ex post del impacto de las regulaciones y la simplificación de trámites y servicios" (Polanco, 2016, pág. 183). Este tema es novedoso, dados los compromisos de control supranacional que contiene, los cuales toman como punto de partida los lineamientos que en esta materia presentan la Organización para la Cooperación y el Desarrollo Económicos (OCDE) y el APEC 5 .

En cuanto a servicios, se establecen reglas de liberalización en los cuatro modos ${ }^{6}$ y se incorporan disciplinas y compromisos tales como las materias de comercio electrónico, telecomunicaciones, servicios financieros y transporte marítimo

Con relación a compras públicas, la AP intentó actualizar los estándares en términos de transparencia, trato nacional y no discriminación, procedimientos de impugnación y uso de medios electrónicos en los procesos de contratación. Con esto, se procura mejorar la publicidad de las convocatorias públicas, para que estas se presenten oportunamente. De igual modo, se enfatiza que las especificaciones técnicas deben ser justas y objetivas, y que la adjudicación de contratos ha de estar basada únicamente en los criterios de evaluación estipulados en los avisos y en las bases de la licitación.

En la medida en que se concrete este escenario de producción de bienes y servicios en la región, se favorecerá el clima de atracción de las inversiones y de capitales productivos para impulsar los negocios internacionales, estimulado por el marco de facilitación del comercio que se viene desarrollando.

Cabe resaltar que, a diferencia del TPP, el Acuerdo Marco de la AP no contiene compromisos en materia de propiedad intelectual, lo que denota que, debido a la sensibilidad del tema, los miembros aún no han considerado asumirlos. Sin perjuicio de ello, la AP ha establecido un grupo de trabajo con miras a armonizar las regulaciones internas en esta materia.

\section{CONCLUSIONES Y DESAFÍOS DE LA ALIANZA DEL PACÍFICO COMO INICIATIVA DE INTEGRACIÓN}

La AP constituye un bloque cada vez más consolidado de países con economías basadas en modelos que propician el libre comercio, que individualmente han negociado acuerdos comerciales con diversas naciones del mundo (incluidas las de Asia-Pacífico, en particular Chile y el Perú) y que, de manera conjunta, representan un gran potencial de oferta exportable. Ello les permitirá, como bloque, aproximarse a los países de Asia-Pacífico aprovechando la posibilidad de establecer cadenas productivas más competitivas (sobre la base, por ejemplo, de las reglas

\footnotetext{
5. En efecto, tanto la OCDE como el APEC han establecido lineamientos generales en cuanto a coherencia regulatoria, que al contener compromisos en esta materia, permiten a su vez contar con medidas transparentes que evitan situaciones de corrupción. Cabe indicar que la OCDE ha realizado un estudio sobre mejora regulatoria para el caso peruano.

6. El Acuerdo General sobre el Comercio de Servicios de la Organización Mundial del Comercio señala que existen cuatro modos de prestación: modo 1, comercio transfronterizo; modo 2 , consumo en el extranjero; modo 3, presencia comercial; y modo 4, presencia de personas físicas.
} 
de origen que las rigen), así como buscar el intercambio comercial de productos y/o servicios complementarios entre los países de ambos bloques.

Si bien es cierto que hubo partes de las negociaciones, tanto de la AP como del TPP, que se hicieron al mismo tiempo, aunque en agendas paralelas (tal fue el caso en el Perú y México), del análisis realizado se puede colegir que el TPP ha servido de base para el esquema de integración profunda asumido en el marco de la AP, pese - como punto adicional - a que no todos los miembros de este última forman parte de aquel.

Aunque los miembros de la AP ya tenían consolidadas zonas de libre comercio, queda claro que el Acuerdo Marco ha permitido ir más allá, con miras a generar cadenas globales de valor, a fortalecer la movilidad de personas, a atraer inversiones y a incursionar en compromisos en materia de coherencia regulatoria.

Parte de las ganancias del Perú en esta iniciativa se encuentran en la oportunidad de estrechar relaciones con México, profundizar las alianzas productivas y sacar provecho de la posición geográfica estratégica. Entre los desafíos del Perú está consolidar esta visión en términos prácticos, para aprovechar las economías de escala y optimizar los procesos productivos, y estrechar así las condiciones para acceder a los mercados asiáticos con grandes oportunidades y convertirse de esta manera en un importante puente comercial entre América del Sur y Asia, al reducir los costos logísticos del comercio exterior.

\section{REFERENCIAS}

Alianza del Pacífico [AP]. (2012). Acuerdo Marco de la Alianza del Pacífico. Recuperado de https:// alianzapacifico.net/?wpdmdl=4464 [Consulta: 2 de marzo de 2017].

Alianza del Pacífico [AP]. (2014). Protocolo Adicional al Acuerdo Marco de la Alianza del Pacífico. Recuperado de https://alianzapacifico.net/?wpdmdl=1327 [Consulta: 2 de marzo de 2017].

Alianza del Pacífico [AP]. (2015). Primer Protocolo Modificatorio del Protocolo Adicional al Acuerdo Marco de la Alianza del Pacífico. Recuperado de https://alianzapacifico.net/?wpdmdl=4580 [Consulta: 2 de marzo de 2017].

Alianza del Pacífico [AP]. (2016). Abecé Alianza del Pacífico 2016. Recuperado de https://alianzapacifico. net/?wpdmdl=4441 [Consulta: 2 de marzo de 2017].

Alianza del Pacífico [AP]. (s. f.). Antecedentes. Recuperado de http://alianzapacifico.net/que-es-laalianza/\#antecedentes [Consulta: 2 de marzo de 2017].
Congreso de la República del Perú. (1993). Constitución Política del Perú. Recuperado de http://portal.jne.gob. pe/informacionlegal/Constitucin\%20y\%20Leyes1/ CONSTITUCION\%2OPOLITICA\%2ODEL\%2OPERU.pdf [Consulta: 15 de abril de 2017].

Foro de Cooperación Económica Asia-Pacífico [APEC]. (1997). Declaración de Vancouver. APEC Ministerial Statement on Membership November 1997 (Approved by the 9th APEC Ministerial Meeting held in Vancouver). Recuperado de https://www.apec.org// / media/Files/MinisterialStatements/Annual/1997/97_ amm_jms.pdf [Consulta: 18 de marzo de 2017].

Foro de Cooperación Económica Asia-Pacífico [APEC], Organización para la Cooperación y el Desarrollo Económicos [OCDE]. (2004). APEC-OECD Integrated Checklist on Regulatory Reform. A Policy Instrument for Regulatory Quality, Competition Policy and Market Openness. Singapur: Autor.

Ministerio de Comercio Exterior y Turismo del Perú. Acuerdos Comerciales del Perú. (2016). El Acuerdo sobre Facilitación del Comercio de la OMC. Recuperado de http://www.acuerdoscomerciales.gob.pe [Consulta: 18 de marzo de 2017].

Ollanta Humala envió el TPP al Congreso. (22 de julio de 2016). Perú21 [Web]. Recuperado de http://peru21.pe/ economia/presidente-ollanta-humala-envio-tpp-alcongreso-2252642 [Consulta: 15 de abril de 2017].

Organización para la Cooperación y el Desarrollo Económicos [OCDE]. (2016). Regulatory Policy in Peru. Assembling the Framework for Regulatory Quality. París: Autor.

Polanco, R. (2016). Convergencia regulatoria en la Alianza del Pacífico: un capítulo inconcluso. Revista de Direito Internacional. Brazilian Journal of International Law, 13(1), 170-195.

Representante de Comercio de los Estados Unidos [USTR]. (2016). III. Bilateral and Regional Negotiations and Agreements. En The President's Trade Policy Agenda. Recuperado de https://ustr.gov/sites/default/files/files/ reports/2016/AP/2016\%20AR\%20Chapter\%203.pdf [Consulta: 18 de marzo de 2017].

Representante de Comercio de los Estados Unidos [USTR]. (2017). I. The President's Trade Policy Agenda. En The President's 2017 Trade Policy Agenda. Recuperado de https://ustr.gov/sites/default/files/files/reports/2017/ AnnualReport/Chapter\%20I\%20-\%20The\%20 President\%27s\%20Trade\%20Policy\%20Agenda.pdf [Consulta: 18 de marzo de 2017]. 